\title{
Discrimination learning with varying numbers of positive and negative stimuli by children of different ages'
}

\author{
Richard D. Walk and Ellen J. Saltz \\ GEORGE WASHINGTON UNIVERSITY
}

\begin{abstract}
Children of 5 to $6 \mathrm{yr}$. and 8 to $9 \mathrm{yr}$. of age were given a two choice discrimination task with either (1) one positive stimulus and two or three negative stimuli (only one of which was used at a time), or (2) two or three positive stimuli and one negative stimulus. Younger children had as many solutions on each type of task while the older Ss were successful only with a single positive stimulus and multiple negative stimuli. Introduction
\end{abstract}

It is well known that adult human Ss learn "positive" concepts easier than they learn "negative" ones (Bruner, Goodnow, \& Austin, 1956; Hovland \& Weiss, 1953). Experimentation on discrimination learning with animal Ss, on the other hand, has shown that animals learn as much from the negative stimulus as from the positive stimulus (Fitzwater, 1952; Moss \& Harlow, 1947).

The task of the present investigation was to try to tie together these disparate lines of research: positive instances in concept formation and the role of the positive and negative stimulus in discrimination learning. Walk (1952) found that adult human Ss would learn a concept discrimination task easier when a single aspect of the stimulus was positive while two parts were negative than when two aspects were positive and one part was negative. A related experiment with rats as Ss (Walk, 1951) found, however, that rats learned equally well in a two choice discrimination task with one positive stimulus and two different negative stimuli as they did with two positive stimuli and a single negative one. The discrimination learning task, unlike the positive concept task, can be used with a variety of organisms.

In the present study children of 5 and $6 \mathrm{yr}$. of age were compared to children of 8 and $9 \mathrm{yr}$. of age. Would younger children learn, like animal Ss, equally well in a two choice discrimination with a single positive stimulus and several negative stimuli as they would with a single negative stimulus and several positive stimuli? Would older children, on the other hand, perform better with one positive stimulus when paired with several negative stimuli than they would with several positive stimuli paired with a single negative stimulus?

\section{Method}

Ss were 78 children 5 to 6 yr. of age and 43 children 8 to $9 \mathrm{yr}$. old. The children were secured from summer day schools in the Washington area.

The apparatus was $43 / 8$ in high and 10 in long, of $3 / 4$ in pine, placed on a base of $3 / 4$ in pine $35 / 8$ in wide. Three vertical pieces of slotted aluminum permitted the insertion of two $41 / 2$ in by 4 in stimulus cards side by side. When $\mathrm{S}$ indicated his choice of one card it was raised by $E$ to expose a $11 / 2$ in hole behind the center of the card which would, if the choice were correct, contain a single $M \& M$ piece of candy. The base behind the hole was surfaced with felt so the reward could be put into place noiselessly. The stimulus cards were of white cardboard with simple orange geometrical figures pasted on them. The figures were a hexagon, a square, a rectangle, a triangle, an inverted $\mathrm{U}$, an inverted $\mathrm{L}$ and an $\mathrm{X}$.

The $S$ was told that one of the two cards would have an $M \& M$ behind it, and that his task was to discover which card or cards had the M\&Ms behind it (them). All Ss were run for 20 trials. Those not reporting a solution, and 10 correct responses in a row, were carried to 30 trials or stopped sooner if a solution occurred.

Experiment I. The positive task used one positive stimulus and paired it with three negative stimuli. During each trial two cards were shown, one positive and one negative card. The same positive stimulus was used on every trial but the negative stimuli varied, randomly, among the three possible stimuli from trial to trial. The negative task was the reverse of this: there were three positive stimuli and only one negative stimulus.

Experiment II. This experiment was similar to Experiment I except that there was one positive (or negative) stimulus and two negative (or positive) stimuli.

Stimuli were chosen from among the forms listed. For every $\mathrm{S}$ run with a given type of positive stimulus and multiple negative stimuli a $\mathrm{S}$ was paired with the same multiple stimuli as positve and the single stimulus as negative. The side of the apparatus chosen for reward on each trial was determined from a table of random numbers.

Preliminary experimentation showed that using two stimuli as positive (or negative) and four stimuli as negative (or positive) could not be solved by children of these ages within 30 trials. Pilot work also showed that 5 and $6 \mathrm{yr}$. old children could easily solve the task presented here when one stimulus was positive and one was negative (12 of 16 Ss solved the problem within 30 trials). 
Table 1. Solutions and Median Errors by the Different Age Groups in the Two Experiments

\begin{tabular}{ccccccccc} 
& & \multicolumn{3}{c}{ Age 5-6 } & \multicolumn{3}{c}{ Age 8-9 } \\
$\begin{array}{c}\text { Experi- } \\
\text { ment }\end{array}$ & Group & N & Solutions & Median & Errors & N & Solutions & Median \\
& Single positive & 18 & $22 \%$ & 13.5 & 17 & $59 \%$ & 5.0 \\
I & Single negative & 18 & $28 \%$ & 15.0 & 16 & $38 \%$ & 12.5 \\
& Single positive & 21 & $33 \%$ & 15.0 & 6 & $83 \%$ & 6.0 \\
II & Single negative & 21 & $19 \%$ & 15.0 & 4 & $25 \%$ & 15.0
\end{tabular}

\section{Results}

The results are shown in Table 1 . For both experiments the older age group with the single positive stimulus was superior to Ss of the same age with a single negative stimulus. On the other hand, younger Ss had as many solutions whether one stimulus was positive or negative. Errors were converted to percentages and the Mann-Whitney test was used to determine differences. The 8 or 9 yr. old group with the single positive stimulus was superior to the same age children with the single negative stimulus $(p<.01)$ and to either younger group. The two groups of younger Ss were not significantly different from each other.

Approximately the same number of solutions was attained with two multiple positive or negative stimuli (Experiment II) as with three multiple positive or negative stimuli (Experiment I).

\section{Diseussion}

The hypothesis that younger Ss would be similar to animal research on the effect of the positive and negative stimulus and older Ss to adult human "positive concept" results was tentatively confirmed, but more research is needed, using more experimental materials, on this problem. Some support for this experiment comes from a recent study by House \& Zeaman (1963) where retardates with MA 4-6 were compared to those with MA 6-8 yr. of age. The "positive compound index" (an index of learning from the positive stimulus object) was significantly higher in the Ss of higher mental age.

The importance of this experiment is in the development of another discrimination learning task where young human Ss may behave similar to animals while older children may behave like adult Ss, a phenomenon already found with transposition (Kuenne, 1946) and with reversal and non-reversal shifts in problem solving
(Kendler \& Kendler, 1962). While the above investigators posited that language behavior favored the older Ss, the present experiment would not seem to point to language as the differentiating factor. Rather, past experience of an undetermined type with reward and punishment would appear to be responsible. Do some cultures, in the words of the popular song, "accentuate the positive," while others stress the "negative"? Are some individuals ( perhaps maladjusted ones, in our culture) more prone to stress negative information? The present experiment may offer a method to study this topic.

References

BRUNER, J. S., GOODNOW, J. J., \& AUSTIN, G. A. A study of thinking. New York: Wiley, 1956.

FITZWATER, M. E. The relative effect of reinforcement and nonreinforcement in establishing a form discrimination. J. comp. physiol. Psychol., 1952, 45, 476-481.

HOUSE, B. J., \& ZEAMAN, D. Miniature experiments in the discrimination learning of retardates. In L. P. Lipsitt \& C. C. Spiker (Eds.), Advances in child development and behavior. Vol 1. New York: Academic Press, 1963.

HOVLAND, C. I., \& WEISS, W. Transmission of information concerning concepts through positive and negative instances. J. exp. Psychol., 1953, 45, 175-182.

KENDLER, H. H., \& KENDLER, T. S. Vertical and horizontal processes in problem solving. Psychol. Rev., 1962, 69, 1-16.

KUENNE, M. R. Experimental investigation of the relation of language to transposition behavior in young children. J. exp. Psychol., 1946, 36, 471-490.

MOSS, E. M., \& HARLOW, H. F. The role of reward in discrimination learning in monkeys. J. comp. physiol. Psychol., 1947, 40, 333-342.

WALK, R. D. The role of concept formation in discrimination learning. Unpublished doctoral dissertation, Harvard University, 1951.

WALK, R. D. Effect of discrimination reversal on human discrimination learning. J. exp. Psychol., 1952, 44, 410-419.

Note

1. This study was performed while Ellen J. Saltz was an undergraduate research participant under NSF GE 1004. 